\title{
Convexal subarachnoid hemorrhage and acute ischemic stroke: a border zone matter?
}

\author{
Alessandro Introna ${ }^{1}$ (D) Domenico Maria Mezzapesa $^{1} \cdot$ Marco Petruzzellis $^{1} \cdot$ Mariantonietta Savarese $^{1}$. \\ Luigi Chiumarulo $^{2} \cdot$ Domenico Sergio Zimatore $^{2} \cdot$ Franca Dicuonzo $^{2} \cdot$ Isabella Laura Simone $^{1}$
}

Received: 24 November 2018 / Accepted: 23 March 2019 / Published online: 2 April 2019

(C) Fondazione Società Italiana di Neurologia 2019

\begin{abstract}
Background Convexal subarachnoid hemorrhage (c-SAH) is an infrequent condition with variable causes. c-SAH concomitant to acute ischemic stroke (AIS) is even less frequent, and the relationship between the two conditions remains unclear.

Methods Between January 2016 and January 2018, we treated four patients who were referred to our stroke unit with ischemic stroke and concomitant nontraumatic c-SAH. The patients underwent an extensive diagnostic workup, including digital subtraction angiography (DSA).

Results All four patients developed acute focal neurological symptoms with restricted MRI diffusion in congruent areas. In three of the patients, infarcts were in a border zone between the main cerebral arteries and c-SAH was nearby. The fourth patient showed a small cortical infarct, and c-SAH was in a border zone territory of the contralateral hemisphere. An embolic source was discovered or strongly suspected in all cases. One patient was treated with intravenous thrombolysis, but this treatment was not related to c-SAH. None of the four patients showed microbleeds or further cortical siderosis, thus excluding cerebral amyloid angiopathy. In addition, DSA did not show signs of vasculitis, reversible cerebral vasoconstriction syndrome, or intracranial arterial dissection.

Conclusions We proposed the embolism or hemodynamic changes of the border zone arterioles as a unifying pathogenetic hypothesis of coexisting c-SAH and AIS.
\end{abstract}

Keywords Embolism $\cdot$ Border zone infarcts $\cdot$ Convexal subarachnoid hemorrhage

\section{Introduction}

Atraumatic convexal subarachnoid hemorrhage (c-SAH) is an unusual presentation of subarachnoid bleeding, typically sparing basal cisterns, ventricles, parenchyma, and interhemispheric fissures [1]. The most common etiologies are cerebral amyloid angiopathy (CAA), reversible cerebral vasoconstriction syndrome (RCVS), and dissection of intracranial arteries, but many other rare causes have been reported [1-4]. In the clinical practice, the etiology remains undetermined in a consistent

Alessandro Introna

ale.introna@gmail.com

1 Neurology Unit, Department of Basic Medical Sciences, Neurosciences and Sense Organs, University of Bari 'Aldo Moro', Piazza Giulio Cesare 11, 70100 Bari, Italy

2 Neuroradiology Unit, Department of Basic Medical Sciences, Neurosciences and Sense Organs, University of Bari 'Aldo Moro', Bari, Italy percentage of cases $(\sim 20 \%)$ [3]. The association of c-SAH and concomitant acute ischemic stroke (AIS) has been increasingly reported and specifically attributed to arterial occlusive disease, CAA, or intracranial cerebral artery dissection [4-11].

\section{Materials and methods}

We retrospectively reviewed all patients admitted to our stroke unit between January 2016 and January 2018 for AIS and found six patients with concomitant c-SAH and AIS. Two patients were excluded from this study because they were not extensively studied (i.e., unable to perform magnetic resonance imaging (MRI)). All patients underwent a diagnostic workup with neuroimaging, including brain computed tomography (CT), MRI with diffusion-weighted imaging (DWI), magnetic resonance angiography (MRA), and gradient recalled echo (GRE) sequences. Digital subtraction angiography (DSA), evaluating both arterial and venous phases, was performed in all but one patient. Oncomarkers and 
autoimmune screening were performed in all patients to rule out neoplastic conditions and systemic vasculitis. Transthoracic echocardiography and 24-h Holter electrocardiogram monitoring were performed to detect cardioembolic sources. Patent foramen ovale ( $\mathrm{PFO}$ ) was investigated through contrast transcranial Doppler (TCD) and transesophageal echocardiography (TEE) in patients with cryptogenic stroke.

This study was approved by the local ethics committee, and all patients gave their written informed consent.

\section{Results}

\section{Patient 1}

A 38-year-old previously healthy male developed left hemiparesis and hemihypesthesia (National Institutes of Health Stroke Scale (NIHSS) score of 4). DWI showed a small cortical lesion in the posterior part of the lenticular nuclei. The patient underwent intravenous thrombolysis with $0.9 \mathrm{mg} / \mathrm{kg}$ recombinant tissue plasminogen activator. Furthermore, 24-h neurological examination was fully normal, and no signs of bleeding were detected on CT scan. At 1 week, the patient complained of a headache without any new neurological symptoms. An MRI scan revealed c-SAH in the right precentral sulcus near an all-new cortical ischemic lesion in the border zone between the right middle cerebral artery (MCA) and the posterior cerebral artery (PCA) (Fig. 1). GRE sequences did not show microbleeds (MB) or cortical siderosis (CS). DSA excluded RCVS, cerebral venous thrombosis (CVT), and other causes of bleeding (Fig. 2). After total reabsorption of blood, clopidogrel was prescribed. In contrast, TCD and TEE showed the presence of PFO, which was eventually closed. At 2-year follow-up, the patient remained asymptomatic (modified Rankin Scale score of 0 ).

\section{Patient 2}

A 73-year-old male had a clinical history of cigarette smoking, hypertension, and ischemic coronary artery disease treated with stenting and hyperlipidemia. He discontinued aspirin and statin treatment because of side effects. Ten days before admission, the patient developed mild fluctuating left-side motor and sensory deficits. His MRI scan showed frontal subarachnoid bleeding, without signs of MBs or CS at GRE images. DWI sequence showed multiple, small areas of restricted diffusion in the right frontal lobe (between the anterior cerebral artery and the MCA territories) and occipital lobe (between the MCA and the PCA territories) (Fig. 1). MRA showed multiple intracranial stenoses of the right MCA and right PCA, confirmed by DSA that excluded vasculitis and CVT (Fig. 2). Unfortunately, follow-up clinical information is not available.

\section{Patient 3}

A 69-year-old male with a history of hypertension and hypercholesterolemia developed a "wake-up" stroke and was therefore admitted with left facial nerve palsy and mild right hemiparesis (NIHSS score of 6). Brain MRI demonstrated DWI-FLAIR mismatch in the right cortical frontal area. Furthermore, FLAIR sequences showed cortical hyperintensity in the contralateral superior frontal sulcus, suggestive of c-SAH (Fig. 1). CAA was excluded because of the absence of MBs and CS on GRE images. DSA did not show signs of vasculitis, RCVS, stenosis of cervical or intracranial vessels, and/or CVT (Fig. 2). An embolic stroke of undetermined source (ESUS) was diagnosed. After 1 week, and total reabsorption of blood, aspirin was initiated.

\section{Patient 4}

A 77-year-old male with a history of hypertension and glucose intolerance developed left hemipostenia 2 days before admission (NIHSS score of 3). CT scan revealed right frontal c$\mathrm{SAH}$, and CT angiography evidenced the presence of an ulcerated fibrolipidic plaque at the origin of the right internal carotid artery (ICA) (Fig. 2). DWI showed a nearby small area of restricted diffusion in the right semi-oval center (Fig. 1, fourth row). GRE images did not show MBs or CS. At day 7, the patient underwent carotid revascularization. At discharge, and after 1 year of follow-up, he was fully asymptomatic.

\section{Discussion}

c-SAH is a rare cause of subarachnoid bleeding, with a low prevalence ranging from 3 to $7.5 \%[1,3,4,6]$. The incidence of c-SAH concomitant with AIS has not been investigated, being only described in larger series collecting only cases of SAH $[3,4,6]$. In our study, exploring the setting of AIS, although limited to 2 years of observation, we identified four cases of coexisting c-SAH and AIS, suggesting a higher frequency than other reports [4-13]. In one large series of c-SAH concomitant with AIS, which followed eight patients over a 6year period, five patients had major artery occlusion/stenosis and three patients fulfilled the criteria for CAA [7]. Generally, the three more reported causes of c-SAH accompanied by AIS were CAA, arterial occlusive disease, and intracranial cerebral artery dissection [7-9].

In elderly patients, CAA is considered to be the most common causes of the simultaneous presence of c-SAH and small brain infarcts, although both c-SAH and infarcts are more often asymptomatic [8]. In our study, we excluded CAA, strictly applying the modified Boston criteria [14]. Nevertheless, in this context, a compelling hypothesis presented itself: because the possible radiological evolution of c-SAH 
Fig. 1 Brain images of convexal subarachnoid hemorrhage and acute ischemic stroke. Each row corresponds to one patient. The first image is a DWI, whereas the second is a gradient echo (patient $1 / 2$ ) or FLAIR (patient 3/4); the third column approximately indicates the localization of AIL and c-SAH. The white circles indicate the AIL, the red ones the c-SAH. The yellow area is vascularized by ACA, the red by MCA, and the blue one by PCA. The vascular territories have great variability inter-individuals. The images of vascular territories are a case courtesy of A. Prof Frank Gaillard. $<\mathrm{a}$ href = "https:// radiopaedia.org/" $>$ Radiopaedia. org $</ a>$. From the case $<\mathrm{a}$ href $=$ "https://radiopaedia.org/cases/ 10814">rID: 10814</a>
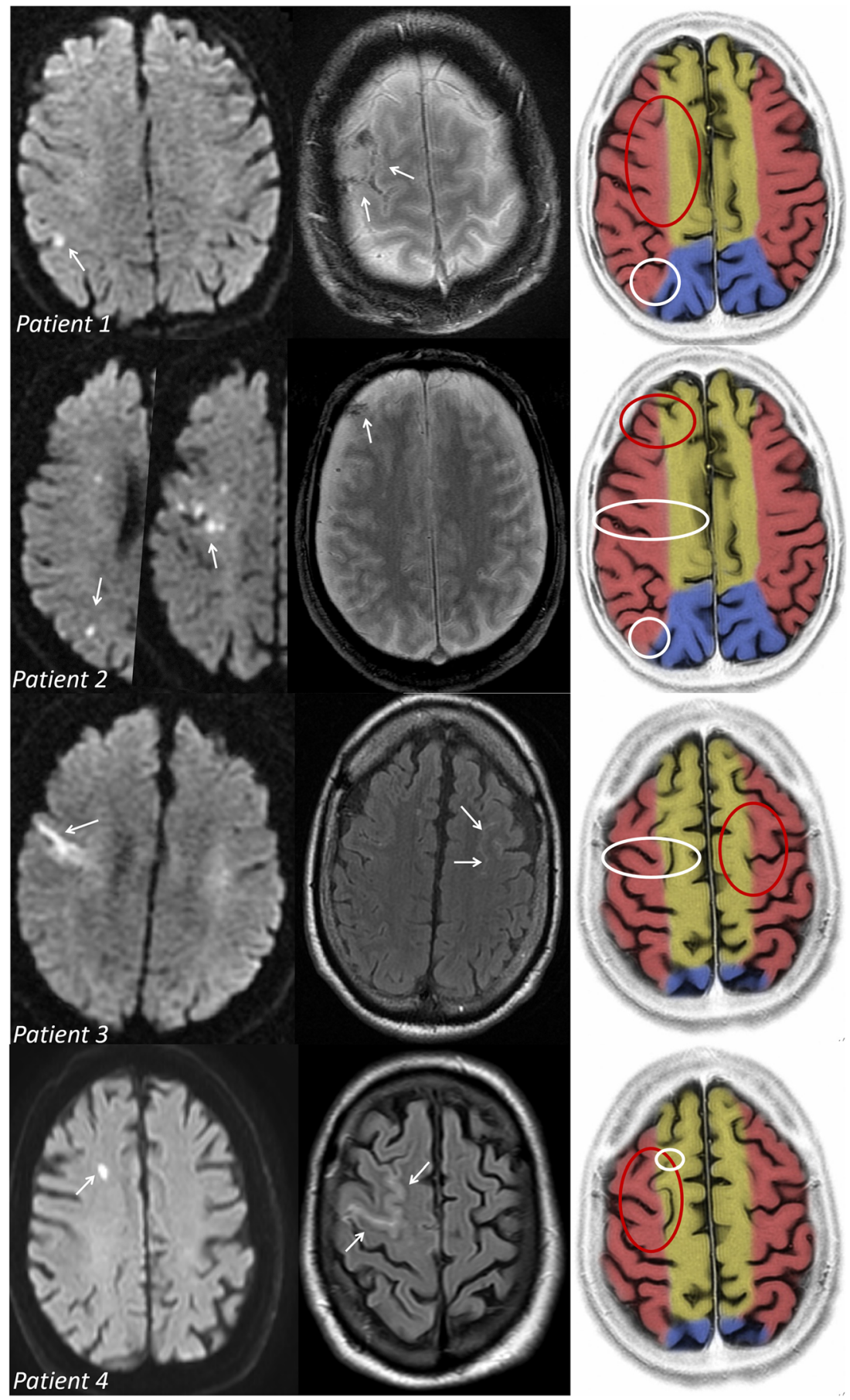

is CS, accounted in CAA diagnosis [14], the mere presence of c-SAH might allow the diagnosis of possible CAA in three of our patients if diagnostic criteria were expanded. A longer follow-up would be necessary to confirm this hypothesis, while the diagnostic work-up revealed other causes.

Our study confirmed an embolic source in three patients, and an embolic source was supposed in one patient. The first patient had a paradoxical embolism from PFO, the second patient had an artery-to-artery embolism from intracranial stenosis, the third patient had an ESUS, and the fourth patient had an embolism from an ulcerated fibrolipidic plaque at the origin of the right ICA. Nakajima and colleagues reported on five patients with occlusion or severe stenosis of major arteries; in two of these patients, they proposed an embolic mechanism for occlusion of large vessels [7]. The main mechanisms of c-SAH were believed to be the rupture of dilated fragile cortical arterioles that were stressed from supplying areas of hypoperfusion and the recanalization of pial vessels, allowing emboli to travel distally. However, probably not all cortical arterioles have the same fragility. 
Fig. 2 Digital subtraction angiography and computed tomography angiography images. Digital subtraction angiography (DSA) of patients 1-3. Patient 1 and 3: DSA (lateral projections of the right and left internal carotid artery, respectively) were totally normal, both in artery and venous phases. Patient 2: white arrow shows stenosis in the right middle cerebral artery. Patient 4: computed tomography angiography of neck vessels shows a defect filling of ICA at its origin (white arrow) for an ulcerate fibrolipidic plaque

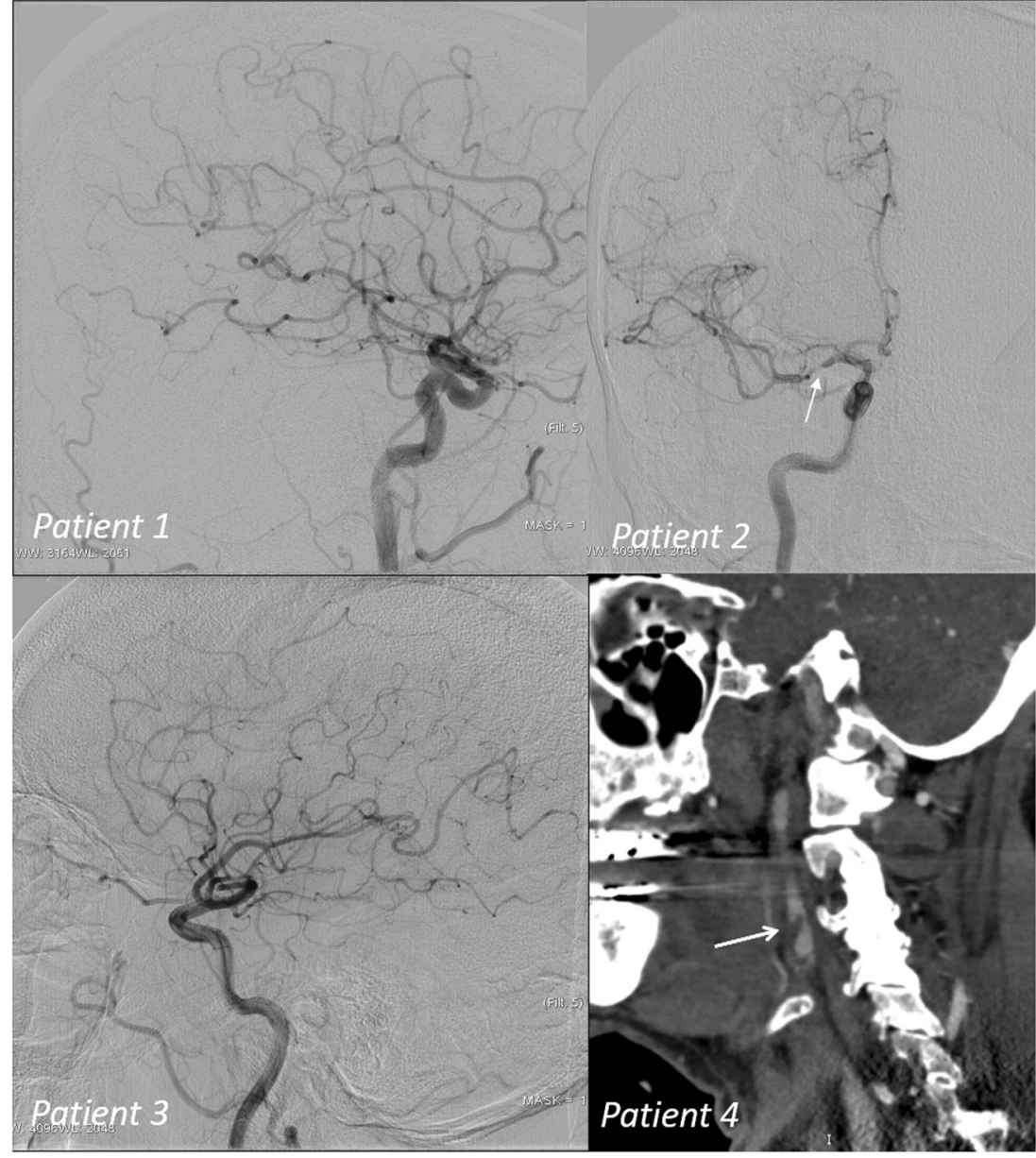

Three of our patients showed cortical border zone infarcts, and one had a small territorial infarct. The etiopathological mechanism of border zone infarcts is not clearly determined and seems to be a mixture of hemodynamic and embolic, more likely hemodynamic for deep locations and embolic for cortical ones [15]. One of the three patients reported on by Usmani and colleagues showed watershed distribution infarction without any vascular disease [5]. Previous reports of AIS concomitant to c-SAH are summarized in Table 1. Reviewing all available images of the cases previously published, the acute ischemic lesions are border zone infarcts, considering the great interindividual variability of cerebral vascular territories.

In addition to infarcts, c-SAH occurred in the arterial border zone in our patients. Interestingly, Fukuma and colleagues found an identical pattern in c-SAH associated with ischemic stroke due to intracranial cerebral artery dissection [9]. Seven percent of strokes due to intracranial dissection developed cSAH with the variable temporal relationship and a hemodynamic change in the border zone arteries was again suggested [9]. Moreover, a similar pattern was detected in patients with high-grade atherosclerotic stenosis of intra- or extracranial cerebral arteries [7]. In these patients, c-SAH was located at the borderline between the dilated and normally perfused territories.

The vulnerability of the distal cortical arterioles wall of the border zone created by an embolism or hemodynamic changes could establish the link between the co-localization of c-SAH and small cortical infarct.

Another element worthy of discussion is that in contrast with a previous report [7], the prognosis was benign in our study. Nakajima and colleagues found a higher rate of CAA in their patients; thus, the poor prognosis was associated with the high incidence of re-bleeding with parenchymal hemorrhage. Our study included a long clinical follow-up for two patients, and they are fully independent. Therefore, patients with coexisting c-SAH and AIS caused by an embolic source had a relatively good outcome even under antithrombotic and anticoagulant therapy and a low risk of re-bleeding.

One limitation of our study is that it had a retrospective design. To better evaluate the incidence and prevalence of cSAH concomitant with AIS, a multicenter prospective study should be performed. Follow-up information is not available for all patients; therefore, conclusions about prognosis must be considered preliminary. 


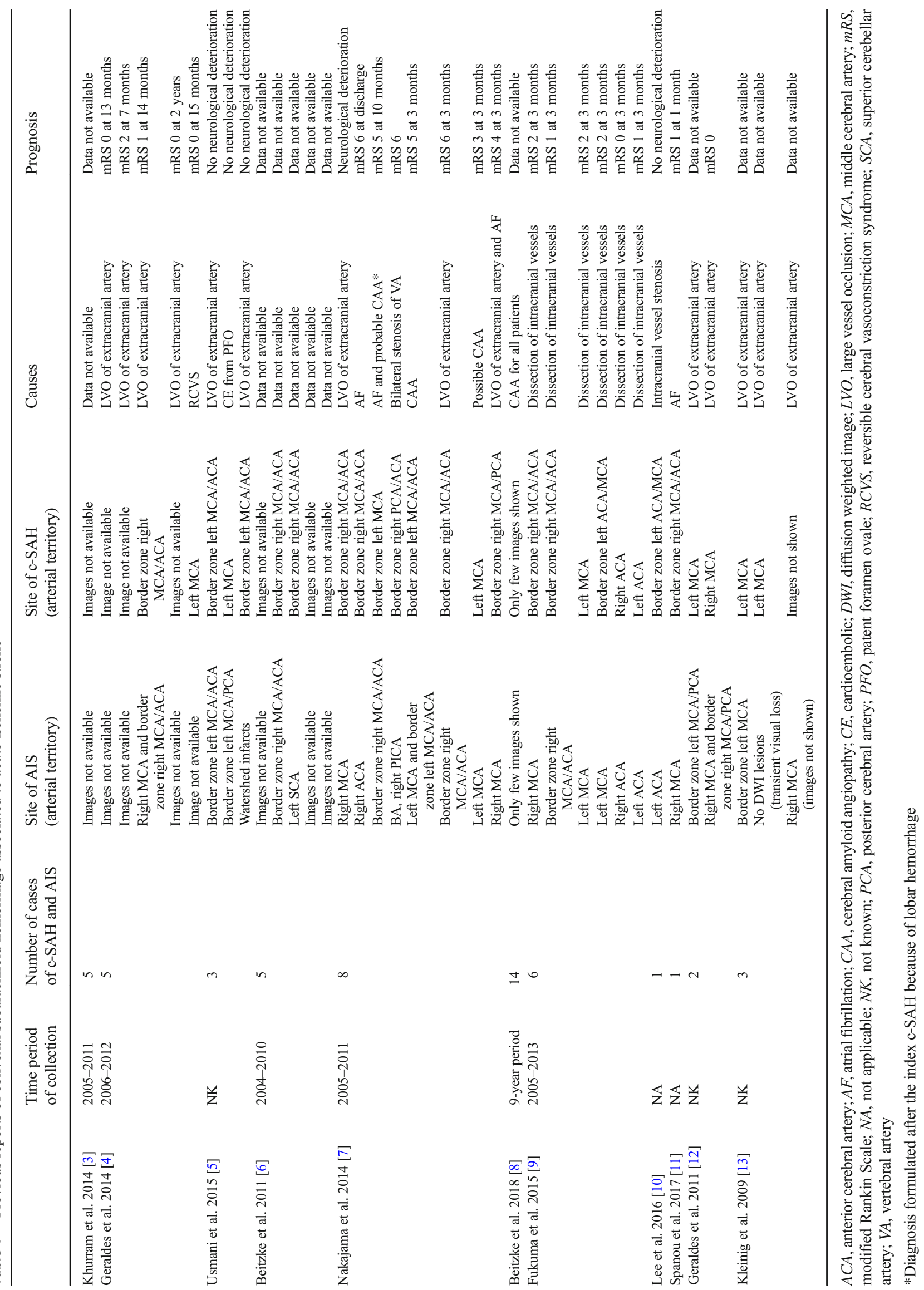




\section{Summary}

The coexistence of c-SAH and AIS may be related to a common etiopathogenetic mechanism that makes the border zone cortical arterioles and brain territories supplied by them more sensible to embolic/hemodynamic insults.

Acknowledgments The authors are grateful to all patients included in this study.

The image of vascular territories was courtesy of Prof. Frank Gaillard. We would like to thank Maria Tappatà, MD, for her help and availability.

Authors' contributions AI, DMM, and MP contributed the conception and design of the study; FD, LC and SZ performed the neuroimages acquisitions and their interpretation; AI wrote the first draft of the manuscript with the equal contribution of DMM and MP and the support of MS.

ILS revised the manuscript for content, including medical writing for content, study concept or design, analysis or interpretation of data, study supervision and coordination.

All of the authors provided final approval of the version to be published and agree to be accountable for all aspects of the work

\section{Compliance with ethical standards}

Statement of ethics This study was approved by the local ethics committee. All patients gave written informed consent.

Conflict of interest The authors declare that they have no conflict of interest.

\section{References}

1. Kumar S, Goddeau RP Jr, Selim MH, Thomas A, Schlaug G, Alhazzani A et al (2010) Atraumatic convexal subarachnoid hemorrhage: clinical presentation, imaging patterns, and etiologies. Neurology 74:893-899

2. Mangla R, Drumsta D, Alamst J, Mangla M, Potchen M (2015) Cerebral convexitysubarachnoid hemorrhage: various causes and role of diagnostic imaging. Emerg Radiol 22:181-195

3. Khurram A, Kleinig T, Leyden J (2014) Clinical associations and causes of convexity subarachnoid hemorrhage. Stroke 45:11511153
4. Geraldes R, Sousa PR, Fonseca AC, Falcão F, Canhão P, Pinho e Melo T (2014) Nontraumatic convexity subarachnoid hemorrhage: different etiologies and outcomes. J Stroke Cerebrovasc Dis 23: e23-e30

5. Usmani N, Ahmad FU, Koch S (2015) Convexity subarachnoid hemorrhage in ischemicstroke. J Neurol Sci 348:259-261

6. Beitzke M, Gattringer T, Enzinger C, Wagner G, Niederkorn K, Fazekas F (2011) Clinical presentation, etiology, and long-term prognosis in patients with nontraumatic convexal subarachnoid hemorrhage. Stroke 42:3055-3060

7. Nakajima M, Inatomi Y, Yonehara T, Hirano T, Ando Y (2014) Nontraumatic convexalsubarachnoid hemorrhage concomitant with acute ischemic stroke. J Stroke Cerebrovasc Dis 23:1564-1570

8. Beitzke M, Enzinger C, Pichler A, Wünsch G, Fazekas F (2018) Acute diffusion-weighted imaging lesions in cerebral amyloid angiopathy-related convexal subarachnoid hemorrhage. J Cereb Blood Flow Metab 38:225-229

9. Fukuma K, Ihara M, Tanaka T, Morita Y, Toyoda K, Nagatsuka K (2015) Intracranial cerebral artery dissection of anterior circulation as a cause of convexity subarachnoid hemorrhage. Cerebrovasc Dis 40:45-51

10. Lee MH, Kim SU, Lee DH, Kim YI, Cho CB, Yang SH et al (2016) Evaluation and treatment of the acute cerebral infarction with convexal subarachnoid hemorrhage. J Cerebrovasc Endovasc Neurosurg 18:271-275

11. Spanou I, Vassilopoulou S, Koroboki E, Tountopoulou A, Velonakis G, Mitsikostas DD (2017) Convexity subarachnoid hemorrhage due to cardioembolic stroke in a woman with thyrotoxicosis: a case report. J Stroke Cerebrovasc Dis 26:e195-e196

12. Geraldes R, Santos C, Canhão P (2011) Atraumatic localized convexity subarachnoid hemorrhage associated with acute carotid artery occlusion. Eur J Neurol 18:e28-e29

13. Kleinig TJ, Kimber TE, Thompson PD (2009) Convexity subarachnoid haemorrhage associated with bilateral internal carotid artery stenoses. J Neurol 256:669-671

14. Linn J, Halpin A, Demaerel P, Ruhland J, Giese AD, Dichgans M et al (2010) Prevalence of superficial siderosis in patients with cerebral amyloid angiopathy. Neurology 74:1346-1350

15. Joinlambert C, Saliou G, Flamand-Roze C, Masnou P, Sarov M, Souillard R et al (2012) Cortical border-zone infarcts: clinical features, causes and outcome. J Neurol Neurosurg Psychiatry 83:771775

Publisher's note Springer Nature remains neutral with regard to jurisdictional claims in published maps and institutional affiliations. 